\title{
The role of PSA density to predict a pathological tumour upgrade between needle biopsy and radical prostatectomy for low risk clinical prostate cancer in the modified Gleason system era
}

\author{
Stavros Sfoungaristos, MD; ${ }^{*}$ loannis Katafigiotis, MD; Petros Perimenis, $M D^{*}$
}

*Patras University Hospital, Urology Department, Greece; 'Laiko University Hospital of Athens, Urology Department, Greece

Cite as: Can Urol Assoc J 2013;7(11-12):e722-7. http://dx.doi.org/10.5489/cuaj.374

Published online November 8, 2013.

\section{Abstract}

Objectives: We evaluate the role of prostate-specific antigen (PSA) density to predict Gleason score upgrade between prostate biopsy material and radical prostatectomy specimen examination in patients with low-risk prostate cancer.

Methods: Between January 2007 and November 2011, 133 lowrisk patients underwent a radical prostatectomy. Using the modified Gleason criteria, tumour grade of the surgical specimens was examined and compared to the biopsy results.

Results: A tumour upgrade was noticed in 57 (42.9\%) patients. Organ-confined disease was found in 110 (82.7\%) patients, while extracapsular disease and seminal vesicles invasion was found in $19(14.3 \%)$ and $4(3.0 \%)$ patients, respectively. Positive surgical margins were reported in $23(17.3 \%)$ patients. A statistical significant correlation between the preoperative PSA density value and postoperative upgrade was found $(p=0.001)$ and this observation had a predictive value $(p=0.002)$; this is in contrast to the other studied parameters which failed to reach significance, including PSA, percentage of cancer in biopsy and number of biopsy cores. Tumour upgrade was also highly associated with extracapsular cancer extension $(p=0.017)$ and the presence of positive surgical margins $(p=0.017)$.

Conclusions: PSA density represents a strong predictor for Gleason score upgrade after radical prostatectomy in patients with clinical low-risk disease. Since tumour upgrade increases the potential for postoperative pathological adverse findings and prognosis, PSA density should be considered when treating and consulting patients with low-risk prostate cancer.

\section{Introduction}

Gleason score (GS) represents a significant histopathological parameter commonly used in nomograms to assay the prognostic outcome of prostate cancer. ${ }^{1}$ As the main surro- gate of cancer aggressiveness, tumour grade informs patient counselling and therapeutic decisions. ${ }^{2}$ In the era of prostatespecific antigen (PSA) screening, more and more patients are primarily diagnosed with insignificant prostate cancer. Since most of these cancers will not become clinically symptomatic, deferred treatment modalities have been introduced to offer similar therapeutic effects while preserving sexual function and continence. ${ }^{3}$ The criteria to define those patients who are suitable to enter conservative treatment protocols are mainly based on PSA, clinical stage, and tumour grade. The latter is the only factor that is not influenced by other pathological entities and consequently represents the most powerful and reliable predictor.

In patients who are finally selected to undergo a surveillance period, physicians have assumed that the tumour grade revealed by the initial diagnostic biopsy represent a true reflection of cancer aggressiveness and determines the patient's low risk for cancer progression. However, many studies have shown that GS obtained by prostate biopsy does not always correlate with final pathological grade of the surgical specimen resected in patients undergoing a radical prostatectomy. ${ }^{4,5}$ In many cases prostate cancer grade is primarily underestimated after the examination of biopsy cores. GS upgrade has been up to $57 \%$ in some studies. ${ }^{4-8}$ The incorrect assessment of tumour grade may lead to inappropriate estimation of cancer aggressiveness and prognosis and finally to the under-treatment of these patients whose cancer potential is higher than that primarily thought.

To understand the negative value of tumour upgrade and the impact on correct treatment selection and prognostic evaluation, the Gleason grading system for prostatic carcinoma underwent its first major revision at the International Society of Urological Pathology (ISUP) consensus conference in 2005. ${ }^{9}$ With this modified Grading system, authors found a shift of the most frequent GS from 6 to $7(3+4)$ in biopsy specimens and an increased degree of agreement between specimens of biopsies and radical prosta- 
tectomies. ${ }^{10}$ Furthermore, the revised Gleason grading may identify more patients with aggressive disease and who have a significantly shorter time to biochemical progression-free outcome after radical prostatectomy. ${ }^{11}$

However, even in the era of the modified Gleason grading system, a significant proportion of patients are under-graded primarily. The main therapeutic and prognostic effect of discordance between biopsy and prostatectomy grade is seen in patients with low-risk disease (GS $\leq 6, \mathrm{PSA}<10 \mathrm{ng} / \mathrm{mL}$, clinical stage T1c-T2a). The main contemporary changes in prostate cancer treatment, in terms of active surveillance and surgical modifications (nerve-sparing, seminal vesiclesparing, lymph node-sparing), have been made in response to the increased rates of over-diagnosis in the PSA era and are mainly applied in patients with low-risk features. In the absence of markers differentiating true low-risk disease from significant disease falsely stratified as low risk, it is very challenging to exclusively select patients who will not experience disease progression. A delay of radical treatment may lead to unfavourable pathological outcomes and poor prognosis.

To evaluate and identify factors low-grade patients by minimizing the upgrade rates, we conducted a prospective analysis of several clinical and pathological variables and we evaluated their potential in upgrade prediction.

\section{Methods}

After we obtained approval by the Ethics committee at our institution, we conducted a prospective analysis of 147 patients with prostate cancer and low-risk disease, as defined by Partin tables. ${ }^{12}$ All patients underwent a retropubic radical prostatectomy between January 2007 and November 2011. All clinical, imaging, laboratory and pathological data were collected and recorded prospectively and analyzed retrospectively.

We excluded patients who had any preoperative therapy for prostate cancer (active surveillance, hormone therapy, radiation therapy) to avoid bias. We also excluded patients with incomplete medical records.

We analyzed preoperative age, preoperative value of PSA, PSA density, number of cores obtained during transrectal ultrasound biopsy procedure, percentage of cancer in biopsy material (PCBM) and the presence of high-grade prostatic intraepithelial neoplasia (HGPIN). We wanted to analyze the association of these factors with upgrading after radical prostatectomy. Any increase in GS between biopsy results and radical prostatectomy was considered as an upgrade. Cases with a biopsy $3+4$ Gleason summary and $4+3$ at radical prostatectomy were not considered an upgrade.

Preoperative PSA was measured before digital rectal examination, transrectal ultrasound or biopsy. Prostate cancer diagnosis was based on transrectal ultrasound biopsy results. During transrectal ultrasound, prostate volume was calculated according to the information of the maximum transverse diameter (D1), the maximum anteroposterior diameter (D2), and the maximum longitudinal diameter (D3) and by using the formula D $1 \times \mathrm{D} 2 \times \mathrm{D} 3 \times$ \% $/ 6$ based on the prostate ellipse dimension theory. PSA density was calculated by dividing the preoperative PSA value and prostate volume.

An open retropubic or laparoscopic extraperitoneal radical prostatectomy was performed in all patients. A histological report concerning tumour grade of the surgical specimen and pathological stage was obtained. The 2009 TNM (Tumour Node Metastasis) classification for prostate cancer was used to classify the pathological stage. We estimated tumour grade after the contemporary criteria of 2005 ISUP modified Gleason system. ${ }^{9}$

Statistical analysis was performed by using SPSS version 17 (SPSS Inc, Chicago, IL). Descriptive statistics are presented as the mean \pm standard deviation (SD) and interquartile range (IQR) for continuous variables and as the absolute and percent frequency for categorical variables. Normality condition of the numerical variables was studied by means of the Kolmogorov-Smirnov test. PSA was the only variable with normal distribution and, consequently, Student's t test was used to compare means. For analyzing the other variables, the Mann-Whitney $U$ test was used and chi-square 2 test was used for categorical ones. Univariate and multivariate analysis was performed to identify the predictive significance of age, preoperative PSA, PSA density, number of biopsy cores, PCBM and the presence of HGPIN. The optimal cutoff values, sensitivity and specificity for quantitative variables, which found to be significant predictors for pathological unilaterality in multivariate analysis, were estimated by using receiver operating curve (ROC) analysis. Positive (true positive/[true positive+false positive]) and negative predictive value (true negative/[true negative+false negative]) were also estimated. All tests were 2 -tailed with $p<0.05$ as statistically significant.

\section{Results}

In total, 147 patients underwent a radical prostatectomy within the study period. From this cohort, 12 were excluded due to insufficient medical records and 2 because they had an operation after a period of active surveillance. Finally, 133 patients met our inclusion criteria and entered our analysis.

The median patient age was 67 years $(65.8 \pm 6.7, \mathrm{SD}$ 11), median preoperative PSA was $7.2 \mathrm{ng} / \mathrm{mL}(7.1 \pm 1.6,2)$ and median PSA density was $0.17 \mathrm{ng} / \mathrm{mL}^{2}(0.19 \pm 0.10 \mathrm{SD}$ 0.13 ). As we previously reported, every patient underwent a transrectal ultrasound biopsy. A 12-core biopsy protocol was applied in $72(54.1 \%)$ patients, while more than 12 
cores were obtained from $61(45.9 \%)$ patients. Based on the pathological results, $22(16.5 \%)$ patients diagnosed with a GS 4 prostate cancer, 24 (18.0\%) had GS 5 and 87 (65.4\%) had 6 . Clinical stage based on digital rectal examination and transrectal ultrasound findings was categorized as follow: 100 (75.2\%) patients with T1c disease and 33 (24.8\%) patients with T2a prostate cancer, respectibely. Following radical prostatectomy, a tumour upgrade was noticed in 57 $(42.9 \%)$ patients. Organ-confined disease was revealed in $110(82.7 \%)$ patients, while cases extracapsular disease and seminal vesicles invasion were found in $19(14.3 \%)$ and 4 $(3.0 \%)$ patients, respectively. Positive surgical margins were reported in $23(17.3 \%)$ patients. Lymph node invasion was found in 2 and both had T3b disease.

We tallied the demographic, clinical and pathologic data regarding the presence of pathological tumour upgrade (Table 1). A statistical significant correlation between the preoperative PSA density value and postoperative upgrade was present $(p=0.001)$. We also tallied pathological outcomes after radical prostatectomy (Table 3). Tumour upgrade was highly associated with extracapsular cancer extension and the presence of positive surgical margins.

In multivariate analysis (Table 3), PSA density was the only variable with statistically significant ability to predict GS upgrade $(p=0.002)$. The estimated optimal cutoff value of PSA density was $0.15 \mathrm{ng} / \mathrm{mL}^{2}$, obtained by ROC analysis (area under the curve 0.643, $p=0.001,95 \% \mathrm{Cl} 0.568$ 0.755). We tally the predictive parameters of PSA density (Table 4).

\section{Discussion}

It is well-established that tumour grade, exported by the Gleason summary, is the most reliable and valuable parameter to estimate the prognosis of prostate cancer. By representing a surrogate of tumour aggressiveness, the grade allows us to stratify patients as either low-, intermediate- or high-risk. It allows informs treatment protocol so that the best oncological outcome is combined with the maximal preservation of the functional results. So far, the calculation of Gleason grade was based on the pathological evaluation of the cores obtained by prostate biopsy. For patients who enter an active surveillance period and close monitoring until disease progression and radical therapy, biopsy results, in combination with PSA levels and clinical stage, are the only data which guide treatment decisions. Furthermore, for patients who undergo a radical prostatectomy, the preservation of the neurovascular bundles and lymph nodes are based solely on the clinical findings and on tumour grade revealed by the biopsy. Nevertheless, the upgrading of the GS between the needle biopsy and radical prostatectomy is not rare. In a recent meta-analysis of 14839 patients, the authors reported a concordance between biopsy and
Table 1. Patient characteristics

\begin{tabular}{|c|c|c|c|}
\hline Characteristics & No upgrade & Upgrade & $p$ value \\
\hline No. patients, n (\%) & $76(57.1)$ & 57 (42.9) & \\
\hline Age (years) & & & $0.868^{\dagger}$ \\
\hline Mean $\pm S D, I Q R$ & $65.7 \pm 6.7,11$ & $66.0 \pm 6.7,10$ & \\
\hline PSA (ng/mL) & & & $0.498^{\S}$ \\
\hline Mean $\pm S D, I Q R$ & $7.0 \pm 1.7,2.4$ & $7.2 \pm 1.6,2.0$ & \\
\hline PSAD (ng/mL²) & & & $0.001^{\dagger *}$ \\
\hline Mean \pm SD, IQR & $0.17 \pm 0.08,0.10$ & $0.22 \pm 0.12,0.14$ & \\
\hline Clinical stage, n (\%) & & & $0.933^{\ddagger}$ \\
\hline $\mathrm{T} 1 \mathrm{c}$ & $58(58.0)$ & $42(42.0)$ & \\
\hline $\mathrm{T} 2 \mathrm{a}$ & $18(54.5)$ & $15(45.5)$ & \\
\hline Biopsy cores, n (\%) & & & $0.960^{\ddagger}$ \\
\hline$\leq 12$ & $41(56.9)$ & $31(43.1)$ & \\
\hline$>12$ & $35(57.4)$ & $26(42.6)$ & \\
\hline РСВМ (\%) & & & $0.750^{\dagger}$ \\
\hline Mean $\pm S D, I Q R$ & $17.2 \pm 16.2,14.3$ & $17.8 \pm 16.9,14.7$ & \\
\hline HGPIN, n (\%) & & & $0.363^{\ddagger}$ \\
\hline No & $30(52.6)$ & $27(47.4)$ & \\
\hline Yes & $46(60.5)$ & $30(39.5)$ & \\
\hline Pathological stage, $r$ & (\%) & & $0.125^{\ddagger}$ \\
\hline $\mathrm{T} 2 \mathrm{a}$ & $12(75.0)$ & $4(25.0)$ & \\
\hline $\mathrm{T} 2 \mathrm{~b}$ & $4(57.1)$ & $3(42.9)$ & \\
\hline $\mathrm{T} 2 \mathrm{c}$ & $52(59.8)$ & $35(40.2)$ & \\
\hline ТЗа & 7 (36.8) & $12(63.2)$ & \\
\hline T3b & $1(25.0)$ & $3(75.0)$ & \\
\hline
\end{tabular}

SD: standard deviation; IQR: interquartile range; PSA: prostate specific antigen; PSAD: PSA density; PCBM: percentage of cancer in biopsy material; HGPIN: high-grade prostatic intraepithelial neoplasia.

prostatectomy grade in $63 \%$, while an upgrade was found in $30 \%$ of patients. ${ }^{13}$ Other higher rates have been published..$^{4-8}$ This observation has important pathological and prognostic aspects. It has been reported that upgraded tumours are significantly larger than tumours which are not upgraded; a significant percentage of them progress outside the prostate capsule. ${ }^{14}$ More specifically, patients with low-grade tumours, meaning that primary biopsy Gleason score $\leq 6$, who are upgraded after radical prostatectomy, are at a significantly higher risk for adverse pathological features and biochemical recurrence than patients who remain with GS $\leq 6$ on final pathology. ${ }^{15}$ Given the fact that conservative treatment protocols and surgical alterations are mainly applied in low-risk patients, these findings are very salient. An underestimation of prostate cancer aggressiveness may lead to under-treatment and, consequently, inappropriate monitoring of biological aggressive tumours. Furthermore, incorrect estimation of GS may lead to the selection of treatments with inferior cure rates, which ultimately adversely affects patient outcome and survival.

To better define the criteria of tumour patterns and minimize the deviation between biopsy and pathological results, more strict criteria were defined. ${ }^{9}$ A modification is that the GS of needle biopsy specimens is rarely $<4$ and that the 


\begin{tabular}{|c|c|c|c|}
\hline & Upgrade & No upgrade & $p$ value \\
\hline Clinical stage, $\mathbf{n}(\%)$ & & & $0.017^{\dagger *}$ \\
\hline$\leq \mathrm{T} 2 \mathrm{c}$ & $42(38.2 \%)$ & $68(61.8 \%)$ & \\
\hline$\geq T 3 a$ & $15(65.2 \%)$ & $8(34.8 \%)$ & \\
\hline Surgical margins, $\mathbf{n}(\%)$ & & & $0.017^{\dagger *}$ \\
\hline Positive & $15(65.2 \%)$ & $8(34.8 \%)$ & \\
\hline Negative & $42(38.2 \%)$ & $68(61.8 \%)$ & \\
\hline Seminal vesicles, $\mathbf{n}(\%)$ & & & $0.187^{\dagger}$ \\
\hline Invasion & $1(25.0 \%)$ & $3(75.0 \%)$ & \\
\hline Tumour-free & 75 (58.1\%) & $54(41.9 \%)$ & \\
\hline
\end{tabular}

most cribriform patterns would be diagnosed as Gleason pattern 4, while only specimens with rare cribriform lesions would be cribriform pattern 3 . The main impact of these alterations is observed in low-grade disease, in which the stratification data are more precise. Consequently, the rates of Gleason upgrade should be decreased. Overall agreement between biopsy and prostatectomy grade improved from $58 \%$ to $72 \%$ by using the ISUP criteria compared to conventional Gleason score. ${ }^{16}$ Furthermore, there is a higher percentage of biopsy specimens with Gleason $\geq 7$ after the ISUP consensus ( $32 \%$ before ISUP vs. $46 \%$ after ISUP) with subsequent decrease in the low-grade tumours $(68 \%$ before ISUP vs. $55 \%$ after ISUP). ${ }^{17}$

The concordance between preoperative and postoperative Gleason scores was recently examined. ${ }^{10}$ The investigators examined the concordance between biopsy and prostatectomy Gleason grade by evaluating the pathologic material either by the standard grading system or either by the modified one. The concordance rates between needle biopsy and radical prostatectomy specimens were $64.1 \%$ when the material was evaluated by conventional grading system and $69.9 \%$ by the modified one. Notably, there was a significant decrease in the upgrading rate when the specimens were evaluated by the modified Gleason grading system $(p=0.026)$, while this significance was obtained when they evaluated the biochemical progression-free survival. Exporting the above results, the authors concluded that the ISUP grading system offers a more reliable way to estimate cancer biological behaviour and to better identify the best candidates for conservative treatments.

There is limited data on the impact of ISUP changes in low-risk patients. In a comparative analysis, $39.6 \%$ of lowgrade patients with needle biopsy GS $\leq 6$ upgraded before ISUP versus $38.1 \%$ to upgrade after ISUP criteria implementation. ${ }^{10}$ Based on our results, there was a higher rate of GS disagreement. Actually, $42.9 \%$ of patients upgraded postoperatively. However, in our analysis we evaluated patients with low-risk disease, not only low-grade tumours, meaning

\begin{tabular}{|c|c|c|c|}
\hline & $p$ value & Odds ratio & $95 \%$ confidence interval \\
\hline Age & 0.833 & 1.006 & 0.955-1.059 \\
\hline PSA & 0.498 & 1.077 & $0.869-1.334$ \\
\hline PSAD & $0.002^{*}$ & 898.429 & $0.119-0.679$ \\
\hline Clinical stage & 0.728 & 1.151 & $0.521-2.541$ \\
\hline Biopsy cores & 0.960 & 1.018 & $0.511-2.028$ \\
\hline PCBM & 0.832 & 1.002 & $0.982-1.023$ \\
\hline HGPIN & 0.363 & 0.725 & $0.362-1.451$ \\
\hline
\end{tabular}

*Statistical significance; PSA: prostate-specific antigen; PSAD: PSA density; PCBM: percentage of cancer in biopsy material; HGPIN: high-grade prostatic intraepithelial neoplasia; Cl: confidence interval.

that some GS $\leq 6$ prostate cancer patients were excluded due to concurrent high-risk features.

It is clear that even in the contemporary era after the modifications in GS estimation, there are many patients with clinically localized disease and low-risk features with highly malignant cancer and with an increased risk of clinical and biochemical progression. The addition of new diagnostic tools would potentially increase the predictive accuracy of clinical stratification and would maximize the therapeutic management of prostate cancer patients. ${ }^{18-21}$

Few studies have evaluated the role of PSA density in upgrade prediction. Corcoran and colleagues have examined the predictive characteristics of PSA density in patients with low- and intermediate-risk disease on biopsy subsequently treated with radical prostatectomy. ${ }^{22}$ They found that $58.3 \%$ of patients with low-grade disease after prostate biopsy upgraded to higher scores and PSA density was a significant predictor $(p<0.001)$ of upgrade in patients with GS $\leq 6$. Similar results were observed by Kojima and colleagues $^{23}$ and Magheli and colleagues, ${ }^{24}$ with $p=0.019$ and $p=0.037$, respectively. Interestingly, PSA density was more accurate than PSA alone to predict Gleason upgrading. When PSA density was added to the predictive model, including PSA and other upgrading predictors, PSA lost its predictive value (OR 1.02, $p=0.423$ ), while PSA density remained an independent predictor (OR 4.89, $p=0.037$ ). Our results add in the above data revealing a beneficial role of PSA density in upgrade prediction $(p=0.002)$, in contrast to the others studied parameters (Table 3 ). A significant association of PSA density to the pathological outcomes follow-

\begin{tabular}{|c|c|}
\hline Sensitivity & $75.4 \%$ \\
\hline Specificity & $42.1 \%$ \\
\hline Positive predictive value & $55.8 \%$ \\
\hline Negative predictive value & $69.6 \%$ \\
\hline
\end{tabular}


ing radical prostatectomy was observed. Gleason upgrade was correlated with increased rates of extracapsular disease and positive surgical margins (Table 2). To be clinically useful, we tried to produce a threshold level of PSA density, over which the possibility of Gleason upgrade increases significantly, triggering either a repeat biopsy or definitive therapy. By using the ROC curve, a cutoff value of $0.15 \mathrm{ng} /$ $\mathrm{mL}^{2}$ was produced. Even though the specificity and positive predictive value were very low, an increased sensitivity and negative predictive value were found (Table 4). Given the complexity of prostate cancer, it seems more effective to use PSA density in preoperative nomograms combined with other predictive factors rather than using it alone.

Our study differs from the other reported studies on 2 levels. Firstly, the evaluation of PSA density predictive ability was adjusted solely for patients with low-risk disease. Secondly, pathological evaluation of the biopsy material and radical prostatectomy specimen were made based on the ISUP modified Gleason criteria. This fact increases the value of the present results since PSA density adds further value to the estimation of tumour grade and aggressiveness.

There are several limitations to our study. The data collection was made prospectively; however, the analysis was made retrospectively. The sample size was relatively small and decreased the strength of the results. On the other hand, these results may be a catalyst for larger, prospective studies. Another limitation is that the PSA density calculation was based on the estimation of prostate volume during the transrectal ultrasound made by 2 different operators. Even though a standard calculation protocol was followed, the inter-observer differences in volume measurement may negatively affect the validity of the results.

\section{Conclusion}

We have determined that PSA density strongly predicts whether a prostate cancer tumour will upgrade between biopsy and radical prostatectomy. Our results may be valuable and can have significant implications in selecting the best treatment protocol for patients for masked aggressive tumours.

Competing interests: Dr. Sfoungaristos, Dr. Katafigiotis and Dr. Perimenis all declare no competing financial or personal interests.

This paper has been peer-reviewed.

\section{References}

1. Partin AW, Mangold LA, Lamm DM, et al. Contemporary update of prostate cancer staging nomograms (Partin Tables) for the new millennium. Urology 2001;58:843-8. http://dx.doi.org/10.1016/S00904295(01)01441-8

2. Gleason DF, Mellinger GT. Prediction of prognosis for prostatic adenocarcinoma by combined histological grading and clinical staging. J Urol 2002;167:953-8. http://dx.doi.org/10.1016/S0022$5347(02) 80309-3$

3. Klotz L, Zhang L, Lam A, et al. Clinical results of long-term follow-up of a large, active surveillance cohort with localized prostate cancer. J Clin Oncol 2010;28:126-31. http://dx.doi.org/10.1200/ JC0.2009.24.2180

4. Steinberg DM, Sauvageot J, Piantadosi S, et al. Correlation of prostate needle biopsy and radical prostatectomy Gleason grade in academic and community settings. Am J Surg Pathol 1997;21:566-76. hittp:// dx.doi.org/10.1097/00000478-199705000-00010

5. Cookson MS, Fleshner NE, Soloway SM, et al. Correlation between Gleason score of needle biopsy and radical prostatectomy specimen: accuracy and clinical implications. J Urol 1997;157:559-62. http:// dx.doi.org/10.1016/S0022-5347(01)65201-7

6. Kvåle R, Møller B, Wahlqvist R, et al. Concordance between Gleason scores of needle biopsies and radical prostatectomy specimens: a population-based study. BJU Int 2009;103:1647-54. http://dx.doi. org/10.1111/.i.1464-410X.2008.08255.x

7. Thickman D, Speers WC, Philpott PJ, et al. Effect of the number of core biopsies of the prostate on predicting Gleason score of prostate cancer. J Urol 1996;156:110-3. http://dx.doi.org/10.1016/ S0022-5347(01)65956-1

8. Corcoran NM, Hong MK, Casey RG, et al. Upgrade in Gleason score between prostate biopsies and pathology following radical prostatectomy significantly impacts upon the risk of biochemical recurrence. BJU Int 2011;108:E202-10. http://dx.doi.org/10.1111/i.1464-410X.2011.10119.x

9. Epstein Jl, Allsbrook WC Jr, Amin MB, et al. The 2005 International Society of Urological Pathology (ISUP) Consensus Conference on Gleason Grading of Prostatic Carcinoma. Am I Surg Pathol 2005;29:1228-42. http://dx.doi.org/10.1097/01.pas.0000173646.99337.b1

10. Uemura H, Hoshino K, Sasaki T, et al. Usefulness of the 2005 International Society of Urologic Pathology Gleason grading system in prostate biopsy and radical prostatectomy specimens. BJU Int 2009;103:11904. http://dx.doi.org/10.1111/i.1464-410X.2008.08197.x

11. Billis A, Guimaraes MS, Freitas LL, et al. The impact of the 2005 international society of urological pathology consensus conference on standard Gleason grading of prostatic carcinoma in needle biopsies. J Urol 2008; 180:548-52. http://dx.doi.org/10.1016/i.juro.2008.04.018

12. Huang $\mathrm{Y}$, Isharwal $\mathrm{S}$, Haese A, et al. Prediction of patient-specific risk and percentile cohort risk of pathological stage outcome using continuous prostate-specific antigen measurement, clinical stage and biopsy Gleason score. BJU Int 2011;107:1562-9. http://dx.doi.org/10.1111/i.1464-410X.2010.09692.x

13. Cohen MS, Hanley RS, Kurteva T, et al. Comparing the Gleason prostate biopsy and Gleason prostatectomy grading system: the Lahey Clinic Medical Center experience and an international meta-analysis. Eur Urol 2008;54:371-81. http://dx.doi.org/10.1016/i.eururo.2008.03.049

14. Fernandes ET, Sundaram CP, Long R, et al. Biopsy Gleason score: how does it correlate with the final pathological diagnosis in prostate cancer? Br J Urol 1997;79:615-7. http://dx.doi.org/10.1046/ j.1464-410X.1997.00126.x

15. Sved PD, Gomez P, Manoharan $M$, et al. Limitations of biopsy Gleason grade: implications for counseling patients with biopsy Gleason score 6 prostate cancer. J Urol 2004;172:98-102. http://dx.doi. org/10.1097/01.ju.0000132135.18093.d6

16. Helpap B, Egevad L. The significance of modified Gleason grading of prostatic carcinoma in biopsy and radical prostatectomy specimens. Virchows Arch 2006;449:622-7. http://dx.doi.org/10.1007/ s00428-006-0310-6

17. Zareba P, Zhang J, Yilmaz A, et al. The impact of the 2005 International Society of Urological Pathology (ISUP) consensus on Gleason grading in contemporary practice. Histopathology 2009;55:384-91. http:// dx.doi.org/10.1111/i.1365-2559.2009.03405.x

18. Dong F, Jones JS, Stephenson AJ, et al. Prostate cancer volume at biopsy predicts clinically significant upgrading. J Urol 2008;179:896-900. http://dx.doi.org/10.1016/i.juro.2007.10.060

19. Gofrit ON, Zorn KC, Taxy JB, et al. Predicting the risk of patients with biopsy Gleason score 6 to harbor a higher grade cancer. J Urol 2007;178:1925-8. http://dx.doi.org/10.1016/i.juro.2007.07.049

20. Pinthus JH, Witkos $M$, Fleshner NE, et al. Prostate cancers scored as Gleason 6 on prostate biopsy are frequently Gleason 7 tumors at radical prostatectomy: implication on outcome. J Urol 2006; 176:979-84. http://dx.doi.org/10.1016/i.juro.2006.04.102

21. Krane $L S$, Menon M, Kaul SA, et al. Role of PSA velocity in predicting pathologic upgrade for Gleason 6 prostate cancer. Urol Oncol 201 1;29:372-7. http://dx.doi.org/10.1016/j.urolonc.2009.04.018 
22. Corcoran NM, Casey RG, Hong MK, et al. The ability of prostate-specific antigen (PSA) density to predict an upgrade in Gleason score between initial prostate biopsy and prostatectomy diminishes with increasing tumour grade due to reduced PSA secretion per unit tumour volume. BJU Int 2012;110:36-42. http:// dx.doi.org/10.1111/j.1464-410X.2011.10681.x. Epub 2011 Nov 15.

23. Kojima $M$, Troncoso $P$, Babaian RJ. Use of prostate-specific antigen and tumor volume in predicting needle biopsy grading error. Urology 1995;45:807-12. http://dx.doi.org/10.1016/50090-4295(99)80088-0

24. Magheli A, Hinz S, Hege C, et al. Prostate specific antigen density to predict prostate cancer upgrading in a contemporary radical prostatectomy series: a single center experience. J Urol 2010;183:126-31. http://dx.doi.org/10.1016/i.juro.2009.08.139
Correspondence: Dr. Stavros Sfoungaristos, Urology resident, Patras University Hospital, Urology Department, Building A, 4th floor, Rion, Patras, 26500 Greece, tel: +302610999367 , fax: +302610993981; sfoungaristosst@gmail.com 